\title{
Reviewers' Responses to Medical Research Articles
}

\author{
Saba Sohail and Jamshed Akhtar
}

\begin{abstract}
Objective: To document the reviewers' responses in terms of reviewers' demographic and professional characteristics, promptness of reply, and duration of reply to the request to review medical research articles for a general biomedical research journal.

Study Design: Cross-sectional, observational study.

Place and Duration of Study: Department of Publications, College of Physicians and Surgeons Pakistan (CPSP), from October to December 2015.

Methodology: Peer reviewed articles edited by a single staff editor were included. Editorials and correspondence were excluded. Manuscript category, discipline, and the total number of reviewers per manuscript were noted. Responses were divided into no response, regrets, and responded, i.e. provided with the review comments; and further sub-divided into timely response, i.e. within 21 days, or later. Total duration of response was counted in days from the date of dispatch to the date of receiving. Among those who provided a review, reviewers' characteristics were noted as designation, institute affiliation, qualification, and gender. Number and percentages of the studied variables were determined. Chi-square test of proportions was used for comparing the proportions with significance at $p<0.05$.

Results: Reviewers for 50 articles including 28 original articles, 15 case reports, three letters to the editor, two short communications, and two new techniques, were evaluated. A total of 598 reviewers were contacted for those 50 articles; forming an average of 11.96 reviewers contacted and 2.2 responded per manuscript. Four hundred and seventy $(78.59 \%)$ did not reply at all, $18(3.01 \%)$ regretted, and $110(18.39 \%)$ responded $(79 / 110=71.81 \%$ timely, and $31 / 110=28.18 \%$ late). Earliest reply was received in one day and the delayed reply in 87 days. Maximum number of reviewers was 24 for a single original article (internal medicine) and 22 for a case report (cardiology). Significantly, more fellows, professors and females $(p=0.004, p=0.002$, and $p=0.017$, respectively) provided timely response.

Conclusion: An overwhelming majority of the reviewers did not reply at all despite the incentives of CME credits and honorarium, adversely affecting the processing time. Majority of those who replied, were on time. Reasons for those who did not reply need to be explored.
\end{abstract}

Key Words: Reviewer, Peer review, Biomedical research, Response.

\section{INTRODUCTION}

Editorial decisions for a research manuscript need technical evaluation for quality assurance and appropriateness of the content, most important of which is the originality of the content. This evaluation checks the archival, statistical, and scientific research contents. While the archival and statistical evaluation is an in-house process carried out by the staff editors and statistician, the expert evaluation of the scientific content takes the form of an expert in the field, who is not a part of the editorial staff or board members. This is called external peer review and its broad aim is to help the editor as a consultant and provide feedback to the author for improvisation of the research communication. 1 By definition, a peer "is a person who is equal in ability, standing, rank or value".2

External peer review process, to be referred subsequently as the Review, at the Journal of College of

Department of Publications, College of Physicians and Surgeons Pakistan (CPSP), Karachi, Pakistan

Correspondence: Prof. Saba Sohail, Department of Publications, College of Physicians and Surgeons Pakistan (CPSP),

$7_{\text {th }}$ Central Street, Phase II, DHA, Karachi, Pakistan

E-mail:drsabasohail@hotmail.com

Received: January 27, 2018; Accepted: September 01, 2018
Physicians and Surgeons (JCPSP) is a double blind prepublication process. ${ }^{3}$ Every manuscript, including the editorials and the letters to editor, reporting a new finding, is subjected to an internal (in-house) and an external peer review. Reviewers at the Journal are selected from a database comprising predominantly of CPSP examiners and supervisors, those who apply for being included in the reviewers' pool and frequent contributors to JCPCP. They must not be the editorial staff of JCPSP or the co-authors of the article under review. They must be academicians with major postgraduate qualification and research background. As a policy matter, JCPSP does not favour suggestions for reviewers by the authors.

For the articles clearing in the initial scrutiny, at least two reviews per manuscript are required - at least one from a technically developed country, before making a definite decision. Every manuscript is initially sent to four reviewers, or more if at least two reviews are not received from those who are initially contacted. The practice of reminders has been withdrawn for the past five years. In line with the paper-free policy of the parent institute, manuscripts are sent to the reviewers by e-mail only. The perks provided to the reviewers are CME credits by CPSP (accredited by the ACCME) and a token honorarium to reviewers residing in Pakistan.

It was repeatedly noted that article processing got delayed by authors and reviewers' non-to-delayed response. ${ }^{3}$ 
The delay in peer review was found to be a very unreliable link in the article processing chain at JCPSP. 4 Timely reviews reduce the processing time; and it is important to identify the non-response patterns to predict as to which reviewers may respond timely.

The rationale of the study was that the characteristics of timely responding reviewers need to be identified. Response patterns need to be evaluated regarding the delay problem to seek remedy to the review problems.

The objective of the study was to document the reviewers' responses in terms of certain reviewers' characteristics, reply and duration of reply to the request to review medical research articles for a general biomedical research journal.

\section{METHODOLOGY}

It was an observational study conducted at the Department of Publications, CPSP, from October to December 2015. All peer reviewed articles, edited by a single staff editor, were included. Editorials and letters to the editor in response to a published articles, were excluded.

Articles were grouped according to the manuscript category, i.e. original articles (OA), case reports (CR), new techniques, surveys etc. The discipline of the specialty and the total number of reviewers per manuscript were noted.

Responses were divided into no response, regrets, and responded, i.e. provided with the review comments. Responses were divided into timely response, i.e. within 21 days, or later, i.e. after 21 days. Total duration of response was counted in days from the date of dispatch to the date of receiving. Those reviewers who did not respond were not included in further analysis. Among those who provided a review, reviewers' characteristics were noted as designation, institute affiliation - public or private - qualification, and gender.

Number and percentages of the studied variables were determined as simple descriptive measures of central tendency and dispersion. Cross-tabulation was done for comparing different variables between the timely and late reviewers groups. Chi-square test was used for comparing proportions of designation, institute affiliation public or private - qualification, and gender among the timely and late reviewers, with significance at $p<0.05$.

\section{RESULTS}

During the three months of study, a total of 50 articles including 28 original articles, 15 case reports, three letters to the editor, two short communications and two new techniques, were edited by a single manuscript editor. A total of 598 reviewers were contacted for those 50 articles forming an average of 11.96 reviewers contacted and 2.2 responded per manuscript. Four hundred and seventy $(78.59 \%)$ did not reply at all, 18
(3.01\%) regretted, and $110(18.39 \%)$ responded $(79 / 110=71.81 \%$ timely, and $31 / 110=28.18 \%$ late $)$. Earliest replies were received in one day for an infectious disease letter to the editor and an ophthalmology case report. The delayed reply took 87 days for a survey from general surgery. The maximum number of reviewers were 24 for a single original article (internal medicine) and 22 for a case report (cardiology).

Qualification of the reviewers showed that 85 had fellowship; 49 (57.6\%) from CPSP (41/49 = 83.6\% being timely reviewers), and 36 from abroad $(17 / 36=47.22 \%$ reviewing timely). There were four $\mathrm{PhDs}$, all reviewing timely. Two reviewers were Diplomat American Board $(D A B)$ and both responded timely. The rest $(n=19)$ had miscellaneous degrees including MPhil, MPHE, MDS; out of whom, 16 (84\%) responded early (Table I).

Academic designations and response trends are shown in Table II, significantly more reviewers of Professorial rank as responding timely. Institutional affiliation and response analysis showed that $70(63.63 \%)$ reviewers belonged to government/public sector institutes. Out of them, $64.28 \%(45 / 70)$ responded within 21 days and $25 / 70(35.7 \%)$ responded later, which was not statistically significant $(p=0.094)$. Forty reviewers belonged to private institutions, out of whom $32(80 \%)$ responded timely and $8(20 \%)$ were late. There were 82 $(74.54 \%)$ male reviewers and $28(25.46 \%)$ female reviewers. Proportionately and significantly more female than male reviewers $(n=25,89.28 \%$ vs. $n=54,65.85 \%$, $p=0.017$ ) provided timely response.

Table I: Qualifications and review response trends of the reviwers.

\begin{tabular}{l|c|c|c|c}
\hline Qualification & Timely & Late response & Total & P-value \\
\hline Fellowship & $57(67 \%)$ & $28(33 \%)$ & 85 & 0.004 \\
MPhil & $15(100 \%)$ & 00 & 15 & \\
PhD & $04(100 \%)$ & 00 & 04 & \\
MDS & 00 & $03(100 \%)$ & 03 & \\
DAB & $02(100 \%)$ & 00 & 02 & \\
MHPE & $01(100 \%)$ & 00 & 01 & \\
\hline
\end{tabular}

Table II: Academic designation and response trends.

\begin{tabular}{l|c|c|c}
\hline Designation (numbers) & Timely response & Late response & p-value \\
\hline Professor (63) & $50(79 \%)$ & $13(21 \%)$ & 0.002 \\
Associate Professor (36) & $18(50 \%)$ & $18(50 \%)$ & \\
Assistant Professor (06) & $06(100 \%)$ & 00 & \\
Consultant (05) & $05(100 \%)$ & 00 & \\
\hline
\end{tabular}

\section{DISCUSSION}

As stressed earlier, an efficient and timely external peer review is a great help to a research editor. This study reiterated the impression that most reviewers do not respond at all, in the process of external peer review.

This difficulty has been felt by the editors in the more developed countries, as well. In most scholarly journals, peer reviewers are usually unpaid, 5 as this service is considered an honor; and a remuneration is not 
considered a due equivalent to their professional service. Moreover, their efforts are also not formally acknowledged. This leads to difficulty in finding competent and efficient reviewers submitting timely reviews, ${ }^{5}$ resulting in publication delays. Nguyen et al. suggested providing incentives to peer-reviewers, it was among the top suggested alterations to the system along with training graduate students in peer-review, increased editorial persistence. 6

It has also been observed that reviewers are more likely to respond positively when the study subject is of their interest. Being pressed for time, is another important factor in declining to review a research study. ${ }^{5}$ It has been suggested that reviewing should be formally recognised by academic institutes; and research journals should formally recognise reviewers' work as an academic contribution of high order of continuing medical education (CME). 5

On an average, a single manuscript was sent to approximately 12 reviewers and two responded for each manuscript in this study. While obtaining response from at least two reviewers and more in case of reviewers' opinion being at variance, is a policy practice at JCPSP. It has been observed by Mutz et al. that increasing the number of reviewers of each manuscript decreases the heterogeneity of response. 7

Contrary to the popular but undocumented belief, a vast majority (65/110) of responders were Professors who replied early, compared to junior ranked academicians and private consultants. This new and hitherto unreported finding nullifies the myth that Pakistani academicians conduct research work only till gaining a certain academic position. In this study, $80 \%$ of the timely reviewers were Professors and two of them replied as early as within a day, which was commendable.

Overall, more reviewers were male, but proportionately more females were earlier responders. However, gender has not been found to affect either the review process or a manuscript's final outcome regarding final acceptance or otherwise. 8

Another new and previously unreported finding of this study was that those affiliated with private set-up responded early in greater proportion (84.61\%) compared to those at public institutes (53.84\%). This effect of institution-affiliation related time-delay response conforms to the prevalent expectations among the Pakistani editors. It may, perhaps, be due to the fact that some private medical universities in Pakistan do provide CME credit for review activities done by their faculty.

Reviewers are extremely important for those journals which do not have a copy editor. 9 They not only improve the scientific content but sometimes bibliography, as well. ${ }^{10}$ Reviewers' comments authenticate observations and the conclusions drawn from them, and help clarify the technical write-up ambiguities, which may not be obvious to a non-specialty editor. It is imperative to enlist their help for making the decision of publishing quality research. 11

Various ways have been suggested to get more involvement of efficient reviewers of high quality. Among them, the most studied are the financial incentives. ${ }^{12-14}$ This may in turn affect the publication cost and revenue of the journal; 15 and some discussions on WAME serve list even argue that this may jeopardise the reviewers' judgment towards submitting speedy reviews without giving a due and thorough evaluation. Since a fair, unbiased and thorough peer review is quintessential for breeding the clinical practice guidelines, ${ }^{16}$ it has to be flawless and genuine, which may get compromised by financial incentives. Awarding CME credits is another suggestion. 12 JCPSP already practises this; the parent institute, that is College of Physicians and Surgeons Pakistan (CPSP), is accredited to award CME points on behalf of the ACCME (American College of Continuing Medical Education).

Another way to get timely and good reviews is to have a sufficiently large pool of reviewers. Selection of reviewers for a research journal depends upon editorial choice, may be subjective and experience-based, and the availability of reviewers for this mostly voluntary activity. ${ }^{17}$ JCPSP, as a policy matter, does not encourage authors to suggest reviewers as this may well open the avenues for publication fraud. This has been described in an interesting detail by Cohen et al. who nipped one such fraud in the bud. ${ }^{18}$

The main limitations of this study are lack of details about those who did not reply. It would have been interesting to know the reasons as to why reviewers did not respond - time constraints, lack of interest, any conflict of research or any other reason.

While peer review is as old as the history of scientific journal publication, ${ }^{19}$ it remains as controversial as it is indispensable. The major strength of this study is that it is a pioneer local attempt to find the reasons which delay the review and ultimately the publication process, in order to find the solution of the problem.

\section{CONCLUSION}

An overwhelming majority of the reviewers did not reply at all despite the incentives of CME credits and honorarium, adversely affecting the processing time. Majority of those who replied, were on time. Reasons for those who did not reply need to be explored.

\section{REFERENCES}

1. Lovejoy TI, Revenson TA, France CR. Reviewing manuscripts for peer-review journals: a primer for novice and seasoned reviewers. Ann Behav Med 2011; 42:1-13.

2. Triggle CR, Triggle DJ. What is the future of peer review? Why is there fraud in science? Is plagiarism out of control? Why do 
scientists do bad things? Is it all a case of: "All that is necessary for the triumph of evil is that good men do nothing"? Vasc Health Risk Manag 2007; 3:39-53.

3. Kurdi MS. Scholarly peer reviewing: The art, its joys and woes. Indian J Anaesth 2015; 59:465-70.

4. Sohail S. Fortifying the external peer review: An editorial perspective. J Coll Physicians Surg Pak 2015; 25:2-3.

5. Tite L, Schroter S. Why do peer reviewers decline to review? A survey. J Epidemiol Community Health 2007; 61:9-12.

6. Nguyen VM, Haddaway NR, Gutowsky LF, Wilson AD, Gallagher AJ, Donaldson MR, et al. How long is too long in contemporary peer review? Perspectives from authors publishing in conservation biology journals. PLoS One 2015; 10:e0132557.

7. Mutz R, Bornmann L, Daniel HD. Heterogeneity of inter-rater reliabilities of grant peer reviews and its determinants: a general estimating equations approach. PLoS One 2012; 7:e48509.

8. Gilbert JR, Williams ES, Lundberg GD. Is there gender bias in JAMA's peer review process? JAMA 1994; 272:139-42.

9. Das D. TH-C-204-02: Improving manuscript quality via structured reviews, enhanced scientific category Taxonomy, and outreach. Med Phy 2016; 43:3868-9.

10. Gasparyan AY, Yessirkepov M, Voronov AA, Gerasimov AN, Kostiyukova EL, Kitas GD. Preserving the integrity of citations and references by all stakeholders of science communication. J Korean Med Sc 2015; 30:1545-52.
11. France CR. Reviewing manuscripts for peer-review journals: a primer for novice and seasoned reviewers. Ann Behav Med 2011; 42:1-13.

12. Ho RC, Mak KK, Tao R, Lu Y, Day JR, Pan F. Views on the peer review system of biomedical journals: an online survey of academics from high-ranking universities. BMC Med Res Methodol 2013; 13:74

13. Garg PK. Financial incentives to reviewers; double-edged sword. J Korean Med Sci 2015; 30:832-3.

14. Gasparyan AY, Gerasimov AN, Voronov AA, Kilas GD. Rewarding peer reviewers: Maintaining the integrity of science communication. J Korean Med Sci 2015; 30:360-4.

15. Diamandis EP. The current peer review system is unsustainable - awaken the paid reviewer force! Clin Biochem 2017; 50:461-3.

16. Vercellini $P$, Buggio L, Vigano $P$, Somigliana E. Peer review in medical journals: beyond quality of reports towards transparency and public scrutiny of the process. Eur $\mathrm{J}$ Int Med 2016; 31:15-9.

17. Gasparyan AY, Kitas GD. Best peer reviewers and the quality of peer review in biomedical journals. Croat Med $J$ 2012; 53: 386-9.

18. Cohen A, Pattanaik S, Kumar P, Bies RR, de Boer A, Ferro A, et al. Organised crime against the academic peer review system. Br J Clin Pharmacol 2016; 81:1012-7.

19. Larson BP, Chung KC. A systematic review of peer review for scientific manuscripts. Hand (NY) 2012; 7:37-44. 